\title{
Gastric and renal effects of COX-2 selective and non-selective NSAIDs in rats receiving low-dose aspirin therapy
}

\section{Marcella Goetz MORO(a) Paula Katherine Vargas SANCHEZ ${ }^{\text {(a) }}$ \\ Mayara Vitorino GEVERT ${ }^{(a)}$ \\ Emeline Maria BALLER ${ }^{(b)}$ \\ Ana Flávia TOSTES(b) \\ Ana Caroline LUPEPSA ${ }^{(b)}$ \\ Sinvaldo BAGLIE(b) \\ Gilson Cesar Nobre FRANCO(c)}

(a) Universidade Estadual de Ponta Grossa - UEPG, Department of Dentistry, Ponta Grossa, PR, Brazil.

(b) Universidade Estadual de Ponta Grossa - UEPG, Department of Pharmaceutical Sciences, Ponta Grossa, PR, Brazil.

(c) Universidade Estadual de Ponta Grossa - UEPG, Department of General Biology, Ponta Grossa, Paraná - Brazil.

Declaration of Interests: The authors certify that they have no commercial or associative interest that represents a conflict of interest in connection with the manuscript.

Corresponding Author: Gilson Cesar Nobre Franco E-mail: gilsoncnf@gmail.com

DOI: 10.1590/1807-3107BOR-2016.vol30.0127

Submitted: Apr 20, 2016

Accepted for publication: Sep 14, 2016

Last revision: Sep 22, 2016

\begin{abstract}
The consumption of low-dose aspirin (LDA) to prevent cardiovascular disease continues to increase worldwide. Consequently, the number of chronic LDA users seeking dental procedures that require complementary acute anti-inflammatory medication has also grown. Considering the lack of literature evaluating this interaction, we analyzed the gastric and renal effects caused by a selective COX-2 inhibitor (etoricoxib) and a non-selective COX-2 inhibitor (ibuprofen) nonsteroidal anti-inflammatory drug (NSAID) in rats receiving chronic LDA therapy. Male Wistar rats were divided into six experimental groups (carboxymethylcellulose (CMC) - vehicle; LDA; LDA + ibuprofen; ibuprofen; LDA + etoricoxib; and etoricoxib) and submitted to long-term LDA therapy with a subsequent NSAID administration for three days by gavage. After the experimental period, we analyzed gastric and renal tissues and quantified serum creatinine levels. The concomitant use of LDA with either NSAID induced the highest levels of gastric damage when compared to the CMC group $(F=20.26, p<0.05)$. Treatment with either LDA or etoricoxib alone was not associated with gastric damage. No significant damage was observed on kidney morphology and function $(F=0.5418, p>0.05)$. These results suggest that even the acute use of an NSAID (regardless of COX-2 selectivity) can induce gastric damage when combined with the long-term use of low-dose aspirin in an animal model. Additional studies, including clinical assessments, are thus needed to clarify this interaction, and clinicians should be careful of prescribing NSAIDs to patients using LDA.
\end{abstract}

Keywords: Anti-Inflammatory Agents; Aspirin; Gastritis; Kidney; Rats.

\section{Introduction}

In dentistry, clinical care of patients who have been chronic users of low-dose aspirin (LDA, 75-325 mg/day) has increased considerably due to the efficacy of this treatment in preventing cardiovascular problems. LDA inhibits platelet aggregation by blocking COX-1, an isoform of the cyclooxygenase enzyme (COX). ${ }^{1}$ Thus, the prescription of short courses of nonsteroidal anti-inflammatory drugs (NSAIDs) after surgical procedures or pathological conditions has become more frequent in patients already taking LDA. ${ }^{2}$ Although, NSAIDs can inhibit both isoforms of COX (COX-1 and COX-2), they are classified as non-selective inhibitors, which act on COX-1 and COX-2 (e.g., ibuprofen), and selective COX-2 inhibitors 
(or coxibs), which are highly selective for the COX-2 isoform (e.g., etoricoxib). ${ }^{3}$

COX-1 is a constitutive isoform responsible for the homeostasis of organs and tissues, and it plays an important role in the gastric mucosa by promoting mucus production and inhibiting acid secretion. ${ }^{4}$ Furthermore, this isoform ensures the maintenance of adequate blood flow in the renal system. ${ }^{5}$ In contrast, COX-2 is typically associated with pathological parameters and promotes healing in the presence of inflammation in the gastric mucosa. ${ }^{4}$ To a lesser degree, this isoform is constitutively expressed in certain organs, including the renal system, where it maintains an adequate glomerular filtration rate and controls blood pressure by physiological modulation of vascular tone and hydric balance in the kidneys. ${ }^{6}$

Studies have shown that the inhibition of COX isoforms by NSAIDs, whether selective or non-selective for COX-2, can lead to minor gastric injuries; however, the inhibition of both COX-1 and COX-2 produces an intensified lesion, suggesting that inhibition of a single isoform is insufficient for the induction of significant damage. ${ }^{78}$ In terms of renal function, the use of NSAIDs alone or in combination can trigger renal alterations, such as fibrosis, necrosis, increased serum creatinine levels, and sodium and water retention. ${ }^{9,10}$

Previous studies have reported the gastric and renal consequences of NSAID use, selective or non-selective and alone or in combination; however, no studies have evaluated the effects of acute use of these drugs in combination with chronic use of LDA. Therefore, we determined the gastric and renal effects caused by the use of a selective COX-2 inhibitor (etoricoxib) and a non-selective inhibitor (ibuprofen) NSAID in rats receiving long-term LDA therapy.

\section{Methodology}

Our experimental protocol followed the ARRIVE Guidelines for Animal Research by the National Centre for the Replacement Refinement \& Reduction for Animals in Research ${ }^{11}$ and was approved by the Ethics Committee of Animal Use at the Universidade Estadual de Ponta Grossa-State University of Ponta Grossa-UEPG-Ponta Grossa/PR, Brazil (protocol CEUA 040/2014). The sample size was calculated using G*Power $3.1^{12}$ software with Type I (a) and Type II ( $\beta$ ) errors of $5 \%$ and $20 \%$, respectively.

Male Wistar rats (10 weeks of age, 250-280 g) were housed in plastic cages (4 animals/ cage) under a 12-hour light/dark cycle at a temperature of $22^{\circ} \mathrm{C}$ with food and water ad libitum. The handling of animals followed the recommendations of the National Council for Animal Experiments Control (CONCEA).

The animals were randomly divided into six groups ( 8 animals/ group). Treatments included long-term (42 days) low-dose aspirin (LDA) and the subsequent administration of a non-selective NSAID (ibuprofen) or a COX-2-selective NSAID (etoricoxib) for three days by gavage (Table).

All drugs were suspended in $0.5 \%$ sodium carboxymethylcellulose (CMC) and administered once a day. To mimic the clinical use of this therapy, NSAIDs were administered two hours after LDA gavage. ${ }^{13}$

Table. Study groups.

\begin{tabular}{|c|c|c|c|}
\hline Group & Treatment & Duration & Reference \\
\hline CMC & $0.5 \%$ sodium carboxymethylcellulose (CMC) - vehicle & 45 days & - \\
\hline LDA & Low-dose aspirin (LDA) $6.75 \mathrm{mg} / \mathrm{kg} /$ day & 45 days & Akyazi et al., 201335; Ghosh et al., $2011^{36}$ \\
\hline \multirow{2}{*}{$\mathrm{LDA}+\mathrm{lbu}$} & $\mathrm{LDA} 6.75 \mathrm{mg} / \mathrm{kg} / \mathrm{day}+$ & 45 days & \multirow{4}{*}{ Liv et al., $2014 ;^{18}$ Bilgin et al., $2013^{19}$} \\
\hline & Ibuprofen 40 mg/kg/day & 3 days & \\
\hline \multirow{2}{*}{ Ibu } & CMC & 42 days & \\
\hline & Ibuprofen $40 \mathrm{mg} / \mathrm{kg} / \mathrm{day}$ & 3 days & \\
\hline \multirow{2}{*}{$\mathrm{LDA}+$ Eto } & LDA 6.75 mg/kg/day + & 45 days & \multirow{4}{*}{ Bressan e Tonussi, 2008;20 Dhull et al., $2012^{21}$} \\
\hline & Etoricoxib $10 \mathrm{mg} / \mathrm{kg} / \mathrm{day}$ & 3 days & \\
\hline \multirow{2}{*}{ Eto } & CMC & 42 days & \\
\hline & Ełoricoxib $10 \mathrm{mg} / \mathrm{kg} /$ day & 3 days & \\
\hline
\end{tabular}




\section{Blood collection and euthanasia}

Under general anesthesia $(90 \mathrm{mg} / \mathrm{kg}$ of $10 \%$ ketamine and $10 \mathrm{mg} / \mathrm{kg}$ of $2 \%$ xylazine, intraperitoneal), blood samples were collected from the abdominal aorta $(3 \mathrm{~mL}) \cdot{ }^{14}$ The animals were euthanized with an overdose of anesthesia.

\section{Gastric macroscopy}

The stomach was removed and opened along the greater curvature to expose the gastric mucosa. This region was rinsed with a $0.9 \%$ saline solution and photographed using a digital camera (Nikon D5300, (C) 2015 Nikon Inc.-Zona Franca de Manaus, Manaus, Brazil). The gastric lesion area was measured $\left(\mathrm{mm}^{2}\right)$ using ImageJ software (National Institute of Mental Health-Bethesda, Maryland, USA), and the results were expressed as a percentage of the total area. ${ }^{15}$

\section{Renal macroscopy}

The kidneys were removed, longitudinally sectioned into two fragments, and photographed using a digital camera (Nikon D5300). We analyzed the cortical and medullary portions for the presence or absence of edema, hemorrhagic foci, and tubular necrosis using previously described methods with some modifications. ${ }^{16}$

\section{Renal function analysis}

As previously described, blood collection was performed immediately prior to euthanasia of the animals to quantify serum creatinine $(\mathrm{Cr})$ levels. Serum was obtained by centrifugation at $735 \times \mathrm{g}$ for 10 minutes, and the $\mathrm{Cr}$ concentration was determined with the colorimetric method using a specific kit (Creatinina WS, Kovalent, Biochemistry, ReageLaborSão Gonçalo, RJ, Brazil) according to the manufacturer's instructions. $^{14}$

\section{Statistical analysis}

Statistical analyses were performed using GraphPad Prism 6 (GraphPad Software Inc.-San Diego, California, USA). The Kolmogorov-Smirnov test was applied to all groups to assess the distribution of data. All values were normally distributed; thus, all statistical analyses were completed using an analysis of variance
(ANOVA) followed by the Tukey's test. The level of significance was set at $5 \%$.

\section{Results}

\section{Gastric evaluation}

The use of LDA (LDA group) and etoricoxib (Eto group) alone did not significantly increase gastric lesions when compared to the vehicle controls (CMC group) $(\mathrm{F}=20.26, \mathrm{p}>0.05)$. In contrast, the administration of ibuprofen (Ibu group) significantly increased the development of gastric lesions. An even higher percentage of injured gastric tissue $(F=20.26$, $\mathrm{p}<0.05)$ was observed in the groups treated with a combination of LDA and ibuprofen (LDA + Ibu group) and LDA and etoricoxib (LDA + Eto group). The effects of each treatment on the gastric mucosa are shown in Figure 1.

\section{Renal evaluation}

Macroscopic and functional renal analyses revealed no statistically significant differences among groups ( $F=0.0, p>0.05$ and $F=0.5418, p>0.05$, respectively). Figure 2 shows the serum creatinine concentration for each experimental group.

\section{Discussion}

In dentistry, short courses of NSAIDs are commonly prescribed to control the pain and inflammation

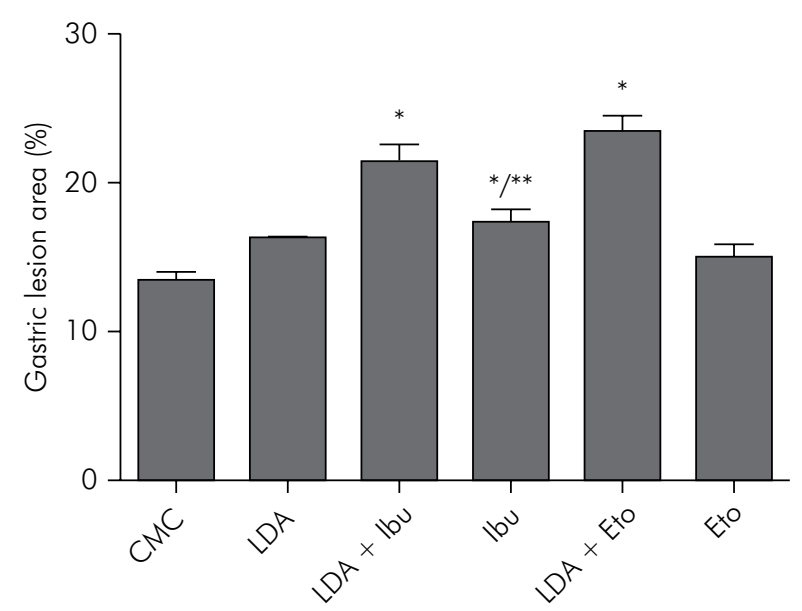

* Statistically significant difference when compared with the CMC group $(p<0.05)$; **Statistically significant difference when compared with the LDA + Ibu and LDA + Eto groups ( $<<0.05)$.

Figure 1. Gastric lesion percentage for each group. 


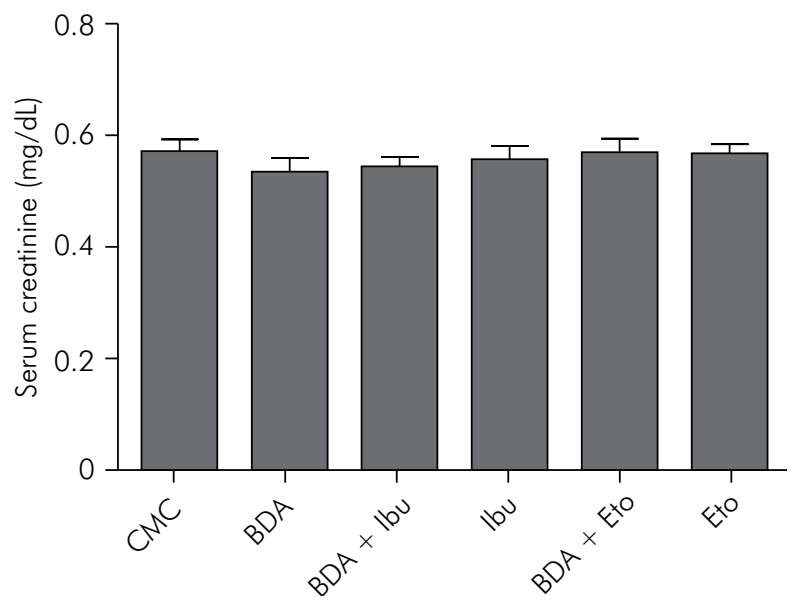

Figure 2. Serum creatinine levels $(\mathrm{mg} / \mathrm{dL})$. There were no statistically significant differences between groups $(p>0.05)$.

related to clinical procedures. In this context, we analyzed the gastric and renal effects of different NSAIDs in rats receiving long-term LDA therapy. NSAID selection was based on their $\mathrm{IC}_{50}$ values, which refers to the measurement of the effectiveness of a substance at inhibiting a specific biological or biochemical function. In this case, a higher $\mathrm{IC}_{50}$ value corresponded to a greater selectivity of the drug for COX-1, whereas a lower $\mathrm{IC}_{50}$ corresponded to a greater selectivity for COX-2. We used the following two NSAIDs for our study: ibuprofen, a non-selective inhibitor $\left(\mathrm{IC}_{50}=15 \mu \mathrm{g} / \mathrm{mL}\right)$, and etoricoxib, a selective COX-2 inhibitor $\left(\mathrm{IC}_{50}=0.0029 \mu \mathrm{g} / \mathrm{mL}\right)^{17}$

The selected doses of ibuprofen $(40 \mathrm{mg} / \mathrm{kg})$ and etoricoxib $(10 \mathrm{mg} / \mathrm{kg})$ were determined with a two-step method: a review of the doses used in previous animal studies that evaluated the anti-inflammatory properties of these drugs, ${ }^{18-21}$ and the calculation of an equivalent dose using the mathematical formula based on body surface area (BSA) suggested by the Food and Drug Administration (FDA). ${ }^{22}$ The doses of ibuprofen and etoricoxib selected for our animal model are equivalent to $400 \mathrm{mg}$ and $100 \mathrm{mg}$ in humans, respectively. These doses are considered to be clinically effective at controlling inflammation and are routinely prescribed by dentists.

A macroscopic analysis was considered sufficient for our current study based on previous reports involving the use of this methodology for observing and quantifying gastric lesions (inflammatory area is calculated as a percentage of the total gastric area) ${ }^{15,23}$ The macroscopic damage observed in rats is considered to be similar to the damage found in humans; furthermore, this damage is typically associated with the clinical symptomatology of gastritis. Macroscopic tissue lesions were not observed in the renal system; therefore, we included the evaluation of serum creatinine levels, which is a key marker of renal function. ${ }^{24}$

To better understand the normal conditions of the gastric mucosa, we analyzed the data from the CMC group. For this comparison, we used naive animals that were not submitted to gavage or any other procedure but rather were simply housed in the vivarium (data not shown). The CMC group showed a gastric lesion area of approximately $12 \%$, and naive animals showed gastric damage of approximately $10 \%(\mathrm{p}>0.05)$.

Interestingly, while the use of etoricoxib alone did not induce gastric lesions, its use in rats receiving LDA resulted in the highest percentage of gastric lesioning. The safety of etoricoxib use alone may be the result of its selective inhibition of COX-2, which consequently preserves the protective COX-1 function in the gastric mucosa. However, prior inhibition of COX-1 (due to LDA administration) combined with the use of a selective COX-2 inhibitor leads to gastric lesions. ${ }^{2,25,26,27}$ Indeed, clinical studies have reported the loss of this "protective effect" of selective COX-2 inhibitors when COX-1 is inhibited by another NSAID. ${ }^{25,26,27}$ The current predicted biological mechanism behind this phenomenon highlights that COX-2 is associated with the healing process, and its inhibition in patients previously subjected to a drug therapy that inhibits COX-1 delayed the normal healing of the mucosa and thus created a higher risk of gastric lesions. ${ }^{2}$

We determined that the isolated use of ibuprofen provoked a significant increase in gastric damage when compared with the CMC group, and the combination of ibuprofen and LDA resulted in an exacerbation of this damage. This effect was likely due a synergic effect of these drugs on the inhibition of COX-1..$^{28}$ In support of our findings, a clinical study conducted with 1,009 patients over two weeks observed a 1.35 relative risk of gastric bleeding in NSAID users, which rose to 3.59 when NSAID use was combined with LDA. ${ }^{29}$ Additionally, another clinical 
study reported the risk of hemorrhage for users of LDA alone, NSAIDs alone, and the combination of LDA and NSAIDs as 3.9, 5.3, and 12.7, respectively. ${ }^{26}$ Thus, we affirm that the concomitant use of LDA and NSAIDs tends to considerably increase the presence of gastric lesions.

Our study also evaluated the effect of LDA on the gastric mucosa. Previous clinical trials suggested this therapy was a risk factor of gastric lesions due to the inhibition of COX-1.30,31 Although we observed increased gastric damage in the LDA group when compared to the CMC group, this difference was not statistically significant. A previous study suggested that the probability of an LDA-induced lesion is directly proportional to the dose administered. Indeed, the probability of developing an injury was approximately doubled in users taking $300 \mathrm{mg} /$ day when compared with users taking $75 \mathrm{mg} /$ day. ${ }^{32}$ In a clinical study performed with daily doses of 100, 100-200, or more than $200 \mathrm{mg}$, patients who took $100 \mathrm{mg}$ /day presented a lower risk of gastric complications when compared with the other doses. ${ }^{33}$ In another trial, long-term users of 75 and $100 \mathrm{mg}$ /day presented a lower incidence of ulcers when compared with doses of 101 to $325 \mathrm{mg} /$ day. ${ }^{34}$ The lack of significance observed in our present study may be associated with the selected LDA dose of $6.75 \mathrm{mg} / \mathrm{kg} /$ day. This dose is equivalent to $75 \mathrm{mg} /$ day in humans, which is the lowest clinical dose used for cardioprotection. ${ }^{35,36}$

We also evaluated the effects of the selected drugs on renal tissue and function. None of the groups showed significant lesions or functional changes. Due to the inhibition of the COX enzyme and, consequently, prostaglandins (PGs), the use of COX-2 selective or non-selective inhibitors may alter

\section{References}

1. Shiotani A, Sakakibara T, Nomura M, Yamanaka Y, Nishi R, Imamura H et al. Aspirin-induced peptic ulcer and genetic polymorphisms. J Gastroenterol Hepatol. 2010;25 Suppl 1:S31-4. doi:10.1111/j.1440-1746.2009.06212.x

2. Kwiecień S, Magierowska K, Śliwowski Z, Wójcik D, Magierowski M, Brzozowski T. New insight into the mechanisms of gastroduodenal injury induced by nonsteroidal anti-inflammatory drugs: practical implications. Pol Arch Med Wewn. 2015;125(3):191-8. the glomerular filtration rate and the regulation of sodium and water. ${ }^{37}$ However, these authors asserted that PGs are typically not the main players in the maintenance of homeostasis in physiological situations but rather only exert their function in instances of previous renal disease. ${ }^{10,38}$

Moreover, when using NSAIDs for a short period of time, the possible hemodynamic alterations in the renal system are reversible; however, long-term and high-dose treatments exacerbate the risk of toxicity. ${ }^{39}$ Thus, although LDA was used for a prolonged period of time, its detrimental effects were reduced by using a lower dose in our study. In contrast, when using ibuprofen and etoricoxib at conventional clinical doses, the risks of renal toxicity were reduced with a short treatment duration. .0,40 $^{0}$

Our research is limited because the use of animal models prohibits the extrapolation of our findings to clinical practice. Additional studies assessing a range of LDA doses are required because the cardioprotective dose of LDA varies from 75 to $325 \mathrm{mg}$.

\section{Conclusion}

Our results suggest that even the acute use of an NSAID (regardless of COX-2 selectivity) can induce gastric damage when combined with the long-term use of LDA in an animal model. Thus, additional studies, including clinical assessments, are needed to clarify this interaction, and clinicians should be mindful of prescribing NSAIDs to patients on an LDA regimen.

\section{Acknowledgments}

This work was supported by the Coordenação de Aperfeiçoamento de Pessoal de Nível Superior (CAPES).

3. Dubois RW, Melmed GY, Henning JM, Bernal M. Risk of upper gastrointestinal injury and events in patients treated with cyclooxygenase (COX)-1/COX-2 Nonsteroidal Antiinflammatory Drugs (NSAIDs), COX-2 Selective NSAIDs, and gastroprotective cotherapy: an appraisal of the literature. J Clin Rheumatol. 2004;10(4):178-89. doi:10.1097/01.rhu.0000128851.12010.46

4. Takeeda M, Hayashi Y, Yamato M, Murakami M, Takeuchi K. Roles of endogenous prostaglandins and cyclooxygenase izoenzymes in mucosal defense of inflamed rat stomach. J Physiol Pharmacol. 2004;55(1 Pt 2):193-205. 
5. Batlouni M. Nonsteroidal anti-inflammatory

drugs: cardiovascular, cerebrovascular and

renal effects. Arq Bras Cardiol. 2010;94(4):556-63.

doi:10.1590/S0066-782X2010000400019

6. Wang J, Xiong X, Feng B. Aspirin resistance and promoting blood circulation and removing blood stasis: current situation and prospectives. Evid Based Complement Alternat Med. 2014;(2014):954863. doi:10.1155/2014/954863

7. Amer M, Bead VR, Bathon J, Blumenthal RS, Edwards DN. Use of nonsteroidal anti-inflammatory drugs in patients with cardiovascular disease: a cautionary tale. Cardiol Rev. 2010;18(4):204-12. doi:10.1097/CRD.0b013e3181ce1521

8. Zinkievich JM, George S, Jha S, Nandi J, Levine RA. Gastric acid is the key modulator in the pathogenesis of non-steroidal anti-inflammatory drug-induced ulceration in rats. Clin Exp Pharmacol Physiol. 2010;37(7):654-61. doi:10.1111/j.1440-1681.2010.05357.x

9. Inal S, Kabay S, Cayci MK, Kuru HI, Altikat S, Akkas $\mathrm{G}$ et al. Comparison of the effects of dexketoprofen trometamol, meloxicam and diclofenac sodium on fibular fracture healing, kidney and liver: an experimental rat model. Injury. 2014;45(3):494-500. doi:10.1016/j.injury.2013.10.002

10. Ungprasert P, Cheungpasitporn W, Crowson CS, Matteson EL. Individual non-steroidal anti-inflammatory drugs and risk of acute kidney injury: a systematic review and meta-analysis of observational studies. Eur J Intern Med. 2015;26(4):285-91. doi:10.1016/j.ejim.2015.03.008

11. Kilkenny C, Browne W, Cuthill IC, Emerson M, Altman DG. Animal research: reporting in vivo experiments: the ARRIVE guidelines. J Cereb Blood Flow Metab. 2011;31(4):991-3. doi:10.1038/jcbfm.2010.220

12. Faul F, Erdfelder E, Lang AG, Buchner A. G*Power 3: A flexible statistical power analysis program for the social, behavioral, and biomedical sciences. Behav Res Methods. 2007;39(2):175-91. doi:10.3758/BF03193146

13. Akagi $Y$, Nio $Y$, Shimada S, Aoyama T. Influence of nonsteroidal anti-inflammatory drugs on the antiplatelet effects of aspirin in rats. Biol Pharm Bull. 2011;34(2):233-7. doi:10.1248/bpb.34.233

14. Khan MA, Neckar J, Cummens B, Wahl GM, Imig JD. Azilsartan decreases renal and cardiovascular injury in the spontaneously hypertensive obese rat. Cardiovasc Drugs Ther. 2014;28(4):313-22. doi:10.1007/s10557-014-6530-0

15. Shin JH, Lee CW, Oh SJ, Yun J, Lee K, Park SK et al. Protective effect of silymarin against ethanol-induced gastritis in rats: role of sulfhydryls, nitric oxide and gastric sensory afferents. Food Chem Toxicol. 2013;55:353-7. doi:10.1016/j.fct.2013.01.019

16. Rodrigues JA, Vanderlei ES, Silva LM, Araújo IW, Queiroz IN, Paula GA et al. Antinociceptive and anti-inflammatory activities of a sulfated polysaccharide isolated from the green seaweed Caulerpa cupressoides. Pharmacol Rep. 2012;64(2):282-92. doi:10.1016/S1734-1140(12)70766-1
17. Patrignani P, Capone ML, Tacconelli S. Clinical pharmacology of etoricoxib: a novel selective COX2 inhibitor. Expert Opin Pharmacother. 2003;4(2):265-84. doi:10.1517/14656566.4.2.265

18. Liu YW, Zhu X, Zhang L, Lu Q, Zhang F, Guo $\mathrm{H}$ et al. Cerebroprotective effects of ibuprofen on diabetic encephalopathy in rats. Pharmacol Biochem Behav. 2014;117:128-36. doi:10.1016/j.pbb.2013.11.027

19. Ozturk Bilgin O, Kumbul Doguc D, Altuntas I, Sutcu R, Delibas N. Effects of subchronic treatment with ibuprofen and nimesulide on spatial memory and NMDAR subunits expression in aged rats. Iran J Pharm Res. 2013;12(4):877-85.

20. Bressan E, Tonussi CR. Antiinflammatory effects of etoricoxib alone and combined with NSAIDs in LPS-induced reactive arthritis. Inflamm Res. 2008;57(12):586-92. doi:10.1007/s00011-008-8029-2

21. Dhull DK, Bhateja D, Dhull RK, Padi SS. Differential role of cyclooxygenase isozymes on neuronal density in hippocampus CA1 region of intracerebroventricular streptozotocin treated rat brain. J Chem Neuroanat. 2012;43(1):48-51. doi:10.1016/j.jchemneu.2011.10.001

22. Reagan-Shaw S, Nihal M, Ahmad N. Dose translation from animal to human studies revisited. FASEB J. 2008;22(3):659-61. doi:10.1096/fj.07-9574LSF

23. Wallace JL, McKnight W, Reuter BK, Vergnolle N. NSAID-induced gastric damage in rats: requirement for inhibition of both cyclooxygenase 1 and 2. Gastroenterology. 2000;119(3):706-14. doi:10.1053/gast.2000.16510

24. Cordaro M, Impellizzeri D, Bruschetta G, Siracusa R, Crupi R, Di Paola R et al. A novel protective formulation of Palmitoylethanolamide in experimental model of contrast agent induced nephropathy. Toxicol Lett. 2016;240(1):10-21. doi:10.1016/j.toxlet.2015.10.006

25. Laine L, Maller ES, Yu C, Quan H, Simon T. Ulcer formation with low-dose enteric-coated aspirin and the effect of COX-2 selective inhibition: a double-blind trial. Gastroenterology. 2004;127(2):395-402. doi:10.1053/j.gastro.2004.05.001

26. Lanas A, Garcia-Rodriguez LA, Arroyo MT, Gomollón F, Feu F, González-Pérez A et al. Risk of upper gastrointestinal ulcer bleeding associated with selective cyclo-oxygenase-2 inhibitors, traditional non-aspirin non-steroidal anti-inflammatory drugs, aspirin and combinations. Gut. 2006;55(12):1731-8. doi:/10.1136/gut.2005.080754

27. Silverstein FE, Faich G, Goldstein JL, Simon LS, Pincus T, Whelton A et al. Gastrointestinal toxicity with celecoxib vs nonsteroidal anti-inflammatory drugs for osteoarthritis and rheumatoid arthritis: the CLASS study: A randomized controlled trial. Celecoxib Long-term Arthritis Safety Study. JAMA. 2000;284(10):1247-55. doi:10.1001/jama.284.10.1247

28. Sørensen HT, Mellemkjaer L, Blot WJ, Nielsen GL, Steffensen FH, McLaughlin JK et al. Risk of upper gastrointestinal bleeding associated with use of low-dose aspirin. Am J Gastroenterol. 2000;95(9):2218-24. doi:10.1111/j.1572-0241.2000.02248.x 
29. Kawasaki K, Kurahara K, Yanai S, Kochi S, Fuchigami T, Matsumoto T. Low-dose aspirin and non-steroidal anti-inflammatory drugs increase the risk of bleeding in patients with gastroduodenal ulcer. Dig Dis Sci. 2015;60(4):1010-5. doi:10.1007/s10620-014-3415-9

30. Nishino M, Sugimoto M, Uotani T, Yamade M, Sahara S, Ichikawa $\mathrm{H}$ et al. Association of gastric mucosal injury severity with platelet function and gastric $\mathrm{pH}$ during low-dose aspirin treatment. Digestion. 2013;88(2):79-86. doi:10.1159/000353150

31. Iwamoto J, Saito Y, Honda A, Matsuzaki Y. Clinical features of gastroduodenal injury associated with long-term low-dose aspirin therapy. World J Gastroenterol. 2013;19(11):1673-82. doi:10.3748/wjg.v19.i11.1673

32. Weil J, Colin-Jones D, Langman M, Lawson D, Logan R, Murphy $\mathrm{M}$ et al. Prophylactic aspirin and risk of peptic ulcer bleeding. BMJ. 1995;310(6983):827-30. doi:10.1136/bmj.310.6983.827

33. Serebruany VL, Steinhubl SR, Berger PB, Malinin AI, Baggish JS, Bhatt DL et al. Analysis of risk of bleeding complications after different doses of aspirin in 192,036 patients enrolled in 31 randomized controlled trials. Am J Card. 2005;95(10):1218-22. doi:10.1016/j.amjcard.2005.01.049

34. Yeomans N, Lanas A, Labenz J, Zanten SV, Rensburg C, Rácz I et al. Efficacy of esomeprazole ( $20 \mathrm{mg}$ once daily) for reducing the risk of gastroduodenal ulcers associated with continuous use of low-dose aspirin. Am J Gastroenterol. 2008;103(10):2465-73. doi:10.1111/j.1572-0241.2008.01995.x
35. Akyazi I, Eraslan E, Gülçubuk A, Ekiz EE, Cırakli ZL, Haktanir D et al. Long-term aspirin pretreatment in the prevention of cerulein-induced acute pancreatitis in rats. World J Gastroenterol. 2013;19(9):2894-903. doi:10.3748/wjg.v19.i19.2894.

36. Ghosh A, Dhumal VR, Tilak AV, Singh A, Pandey M, Bondekar AA. Effect of chronic administration of low dose aspirin on haloperidol induced catalepsy in rats. J Pharmacol Pharmacother. 2011;2(3):198-9. doi:10.4103/0976-500X.83292

37. Ahmetaj-Shala B, Kirkby NS, Knowles R, $\mathrm{Al}^{\prime}$ Yamani M, Mazi S, Wang Z et al. Evidence that links loss of cyclooxygenase-2 with increased asymmetric dimethylarginine: novel explanation of cardiovascular side effects associated with antiinflammatory drugs. Circulation. 2015;131(7):633-42. doi:10.1161/CIRCULATIONAHA.114.011591

38. Dreischulte T, Morales DR, Bell S, Guthrie B. Combined use of nonsteroidal anti-inflammatory drugs with diuretics and/or renin-angiotensin system inhibitors in the community increases the risk of acute kidney injury. Kidney Int. 2015;88(2):396-403. doi:10.1038/ki.2015.101

39. Lomas AL, Grauer GF. The renal effects of NSAIDs in dogs. J Am Anim Hosp Assoc. 2015;51(3):197-203. doi:10.5326/JAAHA-MS-6239

40. Zhang J, Ding EL, Song Y. Adverse effects of cyclooxygenase 2 inhibitors on renal and arrhythmia events: meta-analysis of randomized trials. JAMA. 2006;296(13):1619-32. doi:10.1001/jama.296.13.jrv60015 